Article

\title{
Urinary Sodium Excretion and Blood Pressure Relationship across Methods of Evaluating the Completeness of 24-h Urine Collections
}

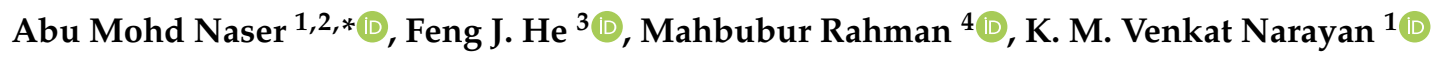 \\ and Norm R. C. Campbell ${ }^{5}$ (D) \\ 1 Emory Global Diabetes Research Center, Hubert Department of Global Health, Rollins School of \\ Public Health, Emory University, Atlanta, GA 30322, USA; KNARAYA@emory.edu \\ 2 Department of Epidemiology, Rollins School of Public Health, Emory University, Atlanta, GA 30322, USA \\ 3 Centre for Environmental and Preventive Medicine, Wolfson Institute of Preventive Medicine, \\ Barts and The London School of Medicine and Dentistry, Queen Mary University of London, \\ London E1 2AD, UK; f.he@qmul.ac.uk \\ 4 International Centre for Diarrhoeal Disease Research, Dhaka 1212, Bangladesh; mahbubr@icddrb.org \\ 5 Department of Medicine, O'Brien Institute of Public Health, Libin Cardiovascular Institute of Alberta at the \\ University of Calgary, Calgary, AB T2N 4Z6, Canada; ncampbel@ucalgary.ca \\ * Correspondence: atitu@emory.edu
}

Received: 26 August 2020; Accepted: 8 September 2020; Published: 11 September 2020

check for updates

\begin{abstract}
We compared the sodium intake and systolic blood pressure (SBP) relationship from complete 24-h urine samples determined by several methods: self-reported no-missed urine, creatinine index $\geq 0.7$, measured 24 -h urine creatinine (mCER) within $25 \%$ and $15 \%$ of Kawasaki predicted urine creatinine, and sex-specific mCER ranges (mCER 15-25 mg/kg/24-h for men; 10-20 mg/kg/24-h for women). We pooled 10,031 BP and 24-h urine sodium data from 2143 participants. We implemented multilevel linear models to illustrate the shape of the sodium-BP relationship using the restricted cubic spline (RCS) plots, and to assess the difference in mean SBP for a $100 \mathrm{mmol}$ increase in 24-h urine sodium. The RCS plot illustrated an initial steep positive sodium-SBP relationship for all methods, followed by a less steep positive relationship for self-reported no-missed urine, creatinine index $\geq 0.7$, and sex-specific mCER ranges; and a plateaued relationship for the two Kawasaki methods. Each $100 \mathrm{mmol} / 24-\mathrm{h}$ increase in urinary sodium was associated with 0.64 (95\% CI: 0.34, 0.94) $\mathrm{mmHg}$ higher SBP for self-reported no-missed urine, 0.68 (95\% CI: 0.27, 1.08) mmHg higher SBP for creatinine index $\geq 0.7,0.87$ (95\% CI: 0.07, 1.67) $\mathrm{mmHg}$ higher SBP for mCER within $25 \%$ Kawasaki predicted urine creatinine, 0.98 (95\% CI: $-0.07,2.02) \mathrm{mmHg}$ change in SBP for mCER within 15\% Kawasaki predicted urine creatinine, and 1.96 (95\% CI: 0.93, 2.99) mmHg higher SBP for sex-specific mCER ranges. Studies examining 24 -h urine sodium in relation to health outcomes will have different results based on how urine collections are deemed as complete.
\end{abstract}

Keywords: blood pressure; sodium; salt intake; urinary sodium; complete 24-h urine

\section{Introduction}

High systolic blood pressure (SBP) is the largest single risk for death globally [1]. High intake of sodium is an important dietary risk factor for high blood pressure (BP) [2]. High-quality epidemiological studies and systematic reviews show strong evidence that high sodium intake causes high BP and there is moderately strong evidence from clinical trials for causing cardiovascular diseases [3-6]. In general, the current diets of populations across the globe contain much higher than recommended sodium $[7,8]$. 
Long-term reduction in sodium intake is one of the most cost-effective strategies to reduce blood pressure and cardiovascular disease [9].

Accurate measurement of daily sodium intake is critical to evaluate the effectiveness of population-based sodium reduction interventions [3]. Sodium measurement in 24-h urine samples is the recommended method to assess daily sodium intake since $93 \%$ of ingested sodium is excreted within 24-h [10]. However, 24-h urine collections are often affected by under- or over-collection, which can result in erroneous estimation of sodium intake [11]. Inclusion of under- or over-collected 24-h urine samples in epidemiological studies may also result in a spurious relationship between sodium intake and health outcomes. Some epidemiological studies use urinary para-aminobenzoic acid (PABA), urinary creatinine, and self-reports for assessment of the completeness of 24-h urine collection [12]. The urinary PABA recovery method is a recommended approach, but it requires participants to take PABA with meals and expensive laboratory procedures [12]. Creatinine-based criteria, such as 24-h urinary creatinine excretion, alone, or with expected urinary creatinine excretion, are often used but can be affected by age, body mass, protein intake, hydration status, and kidney function [11-13]. Comprehensive instruction and having investigators supervise and document the time of the first and last void of 24-h urine collection, and the report of any missed void may be helpful but requires substantial investigator time.

The accuracy and usefulness of creatinine-based criteria to evaluate the completeness of 24-h urine collection has been systematically reviewed [11,12]. However, to our knowledge, there are no studies examining how different methods of assessing the completeness of 24-h urine collections influences the association of urine sodium excretion with health outcomes. We compared the mean sodium intake, and sodium intake and BP relationship from complete 24-h urine samples evaluated by creatinine-based criteria and participants' self-reports using compiled data from Bangladesh.

\section{Materials and Methods}

\subsection{Data Sources}

We compiled data of three cohort studies from Bangladesh, carried out by the International Centre for Diarrhoeal Disease Research, Bangladesh (ICDDR,B). The study protocols were reviewed and approved by the Ethical Review Committee of ICDDR,B (PR-15096 and PR-18004). Informed written consent was obtained from all participants. The studies were predominantly implemented in southwest coastal Bangladesh where communities are exposed to brackish drinking water [14]. In total, we pooled 10,031 person-visits data. Several epidemiological studies from southwest coastal Bangladesh reported sodium intake through drinking water [15-18]. Nevertheless, increased sodium intake through drinking water is seasonal $[19,20]$. In the dry season, communities have limited or no drinking water sources other than brackish groundwater [19]. In contrast, water salinity is not significant during the wet season. Therefore, participants' sodium intake from drinking water was low or minimum during the wet season. The first cohort study followed up 383 participants for two visits (742 person-visits) from southwest coastal Bangladesh during the wet season [21]. The second study was a population-based stepped-wedge randomized controlled trial in southwest coastal Bangladesh that followed up 1190 participants for five monthly visits (5745 person-visits) during the dry season [22,23]. The third cohort study followed up 293 participants from southwest coastal and 277 from non-coastal central Bangladesh for seven visits covering both wet and dry seasons (3547 person-visits data: 1773 from coastal and 1774 from non-coastal regions) [24].

\subsection{Blood Pressure Measurement}

We used a validated Omron ${ }^{\circledR}$ HEM-907 (Kyoto, Japan) digital monitor [19] and an appropriate-sized cuff to measure the BP of the participants between 7 a.m. and 2 p.m. at all visits. Participants did not consume caffeine (e.g., tea, coffee) or food, and did not smoke or perform heavy physical activities $30 \mathrm{~min}$ before BP measurement. Participants rested for at least $5 \mathrm{~min}$ in a chair with back support in the 
sitting position with the arm supported at heart level. BP was measured three times and the mean BP was used for analyses.

\subsection{Demographic, Cardiovascular Risk Factor, and Physical Measures Data}

We collected participants' demographics (age, sex, and religion) information, and their self-reported data on smoking, work-related physical activities, alcohol consumption, and sleep hours during all visits. Participants' weight was measured in all visits, but height was measured once. To calculate households' wealth, we collected information on households' ownership of refrigerators, televisions, mobile phones, motorcycles, bicycles, sewing machines, chairs, tables, wristwatches, wardrobe wooden cots, motor pumps, rice husking machines, motorized rickshaw, cars, and access to electricity [25].

\subsection{4-h Urinary Sodium and Creatinine}

We collected participants' 24-h urine at all visits. Each participant received a 4-L container for urine collection, and a small container to transfer the voided urine to the 4 - $\mathrm{L}$ container. Participants were instructed not to collect their 1st-morning urine and to start urine collection by collecting and transferring the 2nd-morning void, and all voids up to and including the next morning's 1st void. In the second and third cohort studies, participants or a family member who could read or write received a small questionnaire to record the times of the first and last void, and to report any missed voids. Field research assistants recorded the collected 24 -h urine volume and then obtained a 15-mL urine sample from the 4-L container after stirring. They transported the urine samples to a field laboratory at $2-8{ }^{\circ} \mathrm{C}$. Direct ion-selective electrode (ISE) methods were used to measure the urinary sodium with a semi-auto electrolyte analyzer (Biolyte2000, Bio-care Corporation, Taiwan, coefficient of variation: $+5 \%)$. Creatinine was measured by the Jaffe reaction [26].

\subsection{Methods of Evaluating the Completeness of 24-h Urine Collections}

We determined the completeness of 24-h urine collection based on self-reported information and creatinine-based methods. Two self-reported methods were considered: (1) Participants reported no missed voids, and (2) a 22-26 h interval between the time of the first and last urine void plus no missed voids. For the 2 nd self-reported method, we standardized the urine volume for $24 \mathrm{~h}$. We considered four creatinine-based methods: creatinine index $\geq 0.7$ [12], measured 24-h urine creatinine (mCER) within $15 \%$ of Kawasaki predicted daily urine creatinine excretion [27], mCER within 25\% of Kawasaki predicted daily urine creatinine excretion, and sex-specific mCER ranges (mCER 15-25 mg/kg/24 h for men and 10-20 mg/kg/24 h for women) [12,28]. Person-visits that reported any self-reported missed voids or incomplete urine collections were excluded from all creatinine-based methods. The creatinine index was defined as measured 24 - $h$ urine creatinine in $\mathrm{mg} /(21 \times$ bodyweight $)$ for women and measured 24-h urine creatinine in $\mathrm{mg} /(24 \times$ bodyweight $)$ for men [13]. The Kawasaki predicted daily urine creatinine excretion was calculated using the Kawasaki formula: $-12.63 \times$ age $+15.12 \times$ weight +7.39 $\times$ height -79.90 for men, and $-4.72 \times$ age $+8.58 \times$ weight $+5.09 \times$ height -74.50 for women [29] .

\subsection{Statistical Analyses}

We derived the household wealth score by the principal component analysis using the household asset ownership data [24]. We then categorized the wealth score into wealth quintiles. We reported the mean and standard deviation or median and interquartile range, where appropriate, for continuous variables. We reported the proportion of the categorical covariates. We used the Kernel density plots to compare the distributions of urine sodium excretion by the different methods, and the two-sample t-test for evaluating the difference in mean urine sodium excretions across the methods.

In a post hoc analysis, using data from all methods of assessing complete 24-h urine samples, we plotted the restricted cubic spline [30] to examine the trajectories of systolic and diastolic BP with increasing levels of urinary sodium excretion. The restricted cubic spline plots were initially created for men's and women's person-visits combined, and then separately. We used default four knots at 
5th, 35th, 65th, and 95th percentiles to create the restricted cubic spline plots [31], after running the multilevel linear models with the participant-, household-, and community-level random intercepts to account for the clustering of data at different levels. We estimated the models using the maximum likelihood approach and clustered robust standard errors. We adjusted the models for age, sex, and body mass index (BMI), smoking and alcohol consumption, physical activity, religion, hours of sleep, and household wealth. Religion was considered as a covariate since the dietary habits across the two prominent religions are different in our study areas: Hindus are often vegetarian, but Muslims consume meat [32]. Sleep duration was considered a covariate because it can influence BP [33]. We did the Winsorization of the urine sodium at 0.2 th and 99.8 th percentiles to avoid distortion of the restricted cubic splines by the extreme urine sodium values at both tails of the distribution [34]. Winsorization replaced all values before the 0.2 th percentile and after the 99.8 th percentiles with the specific values of those percentiles.

We also modeled urinary sodium as a categorical variable using tertiles of urinary sodium. Similar multilevel linear models to those described above were used to determine the associations of quartile 2, and 3 urinary sodium with BP compared to quartile 1 . We reported the $p$-value for the linear trend determined by the orthogonal polynomials [35]. Since we identified a linear trend for the urine sodium-SBP relationship in tertile analyses, we also assessed the difference in mean blood pressure associated with 100-mmol increases in 24-h urine sodium (continuous exposure) using the similar multilevel linear regression described above. For the tertile and continuous urine sodium analyses, we reported the findings of unadjusted models; models adjusted for age, sex, and body mass index (BMI); and models additionally adjusted for smoking and alcohol consumption, physical activity, religion, hours of sleep, and household wealth. All analyses were conducted in STATA version 16.0.

\section{Results}

Of the total of 9804 person-visits with 24-h urine collections, 7176 (73\%) reported no missed voids, and 5524 (56\%) reported $22-26 \mathrm{~h}$ of urine collection and no missed voids (Table 1 ). The creatinine index $\geq 0.7$ included 5995 (61\%) person-visits, the mCER within $15 \%$ of Kawasaki predicted creatinine included $1569(16 \%)$ person-visits, the mCER within $25 \%$ of Kawasaki predicted creatinine included $2439(25 \%)$ person-visits, and the sex-specific and mCER range included 2109 (22\%) person-visits (Table 1).

In total, $96 \%$ of the samples selected by mCER within $15 \%$ of Kawasaki predicted urine creatinine, and $94 \%$ of samples selected by mCER within $25 \%$ of Kawasaki predicted urine creatinine were also included by creatinine index $\geq 0.7$. All $(100 \%)$ of the samples selected by mCER within $15 \%$ of Kawasaki predicted urine creatinine were also selected by mCER within $25 \%$ of Kawasaki predicted urine creatinine. In total, $73 \%$ of the samples included by the sex-specific range were included by creatinine index $\geq 0.7$, and $67 \%$ included by mCER within $15 \%$ of Kawasaki predicted urine creatinine were included by the sex-specific $\mathrm{mCER}$ range.

The mean age of the person-visits was the highest ( $44.3 \pm 14.6$ years) for the sex-specific mCER ranges, and the lowest ( $42.3 \pm 14.2$ years) for the creatinine index $\geq 0.7$ criteria (Table 1$)$. The proportion of male person-visits included in the complete 24-h urine collection subsample was $30 \%$ for both Kawasaki formula-based methods, 31\% for the sex-specific mCER ranges, and 39\% for all person-visits. The mean BMI was the highest for the sex-specific mCER ranges $\left(23.3 \pm 4.1 \mathrm{~kg} / \mathrm{m}^{2}\right)$ and the lowest $\left(22.4 \pm 4.1 \mathrm{~kg} / \mathrm{m}^{2}\right)$ for the creatinine index $\geq 0.7$ criteria (Table 1$)$. 
Table 1. Age, sex, and BMI distributions of person-visits in subsamples of the study population captured by different methods of evaluating the completeness of 24-h urine samples.

\begin{tabular}{|c|c|c|c|c|c|c|c|}
\hline Characteristics & $\begin{array}{l}\text { All Person-Visits } \\
\text { of 24-h Urine } \\
\text { Collected }\end{array}$ & $\begin{array}{l}\text { Self-Reported No } \\
\text { Missed Voids }\end{array}$ & $\begin{array}{l}22-26 \text { h Urine } \\
\text { Samples with No } \\
\text { Missed Voids }\end{array}$ & $\begin{array}{c}\text { Creatinine Index } \\
\geq 0.7 \text { \& No Missed } \\
\text { Voids }\end{array}$ & $\begin{array}{c}\text { mCER within } 15 \% \\
\text { of Kawasaki } \\
\text { pCER and No } \\
\text { Missed Voids }\end{array}$ & $\begin{array}{c}\text { mCER within } 25 \% \\
\text { of Kawasaki } \\
\text { pCER and No } \\
\text { Missed Voids }\end{array}$ & $\begin{array}{c}\text { mCER } 15-25 \mathrm{mg} / \mathrm{kg} / 24 \mathrm{~h} \\
\text { (Men) and } \\
10-20 \mathrm{mg} / \mathrm{kg} / 24 \mathrm{~h} \\
\text { (Women), and } \\
\text { No Missed Voids }\end{array}$ \\
\hline Person-visits included, N (\%) & $9804(100 \%)$ & $7176(73 \%)$ & $5524(56 \%)$ & $5995(61 \%)$ & $1569(16 \%)$ & $2439(25 \%)$ & $2109(22 \%)$ \\
\hline Age, mean (SD) & $42.7(14.4)$ & $42.8(14.5)$ & $42.8(14.4)$ & $42.3(14.2)$ & $42.9(14.6)$ & $43.0(14.5)$ & $44.3(14.6)$ \\
\hline \multicolumn{8}{|c|}{ Age category, \% (n) } \\
\hline $20-<30$ years & $20(1974)$ & $20(1490)$ & $20(1110)$ & $21(1300)$ & $18(295)$ & $18(457)$ & $16(342)$ \\
\hline $30-<40$ years & 29 (2829) & $28(2093)$ & $28(1560)$ & 29 (1777) & $30(484)$ & $30(731)$ & $29(605)$ \\
\hline $40-<50$ years & 20 (1937) & $20(1468)$ & 20 (1109) & $20(1270)$ & $18(286)$ & $18(456)$ & $19(392)$ \\
\hline $50-<60$ years & 16 (1617) & 16 (1224) & 17 (909) & 16 (1003) & $15(249)$ & $16(391)$ & 19 (391) \\
\hline $60-<70$ years & $10(1006)$ & $11(785)$ & $11(604)$ & $10(630)$ & $12(186)$ & $12(303)$ & $13(274)$ \\
\hline$\geq 70$ years & $5(441)$ & $5(372)$ & $4(239)$ & $4(275)$ & $7(108)$ & $6(140)$ & $5(105)$ \\
\hline Male sex, \% (n) & $39(3802)$ & $36(2677)$ & $34(1893)$ & $35(2198)$ & $30(473)$ & $30(742)$ & $31(664)$ \\
\hline BMI, mean (SD) & $22.4(3.9)$ & $22.5(4.1)$ & $22.6(3.8)$ & $22.4(4.1)$ & $22.9(4.0)$ & $22.9(4.0)$ & $23.3(4.1)$ \\
\hline \multicolumn{8}{|c|}{ BMI categories, $\%$} \\
\hline Underweight & 15 (1445) & $14(1070)$ & $14(748)$ & $15(910)$ & $14(218)$ & $13(331)$ & $11(227)$ \\
\hline Normal weight & $43(4251)$ & 43 (3169) & 42 (2309) & $43(2674)$ & 38 (609) & $39(963)$ & $39(821)$ \\
\hline Overweight & $31(3081)$ & $32(2348)$ & $33(1852)$ & 32 (1995) & $34(550)$ & $34(846)$ & $35(740)$ \\
\hline Obese & $11(1027)$ & $11(854)$ & $11(622)$ & $11(676)$ & $14(231)$ & $14(338)$ & $15(321)$ \\
\hline $\begin{array}{l}\text { 24-h urine Na for both sexes, } \\
\text { mean(SD) }\left[p \text {-value }{ }^{¥}\right]\end{array}$ & $155(76)[0.011]$ & 158 (76) [reference] & $162(76)[0.003]$ & $169(74)[<0.001]$ & $151(79)[<0.001]$ & $152(74)[<0.001]$ & $137(66)[<0.001]$ \\
\hline $\begin{array}{l}\text { 24-h urine Na for males, } \\
\left.\text { mean(SD) [ } p \text {-value }{ }^{¥}\right]\end{array}$ & 149 (79) [0.003] & 155 (81) [reference] & $161(84)[0.016]$ & $169(80)[<0.001]$ & $151(99)$ [0.344] & $151(87)$ [0.246] & $133(57)[<0.001]$ \\
\hline $\begin{array}{l}\text { 24-h urine } \mathrm{Na} \text { for females, } \\
\text { mean(SD) }\left[p \text {-value }{ }^{\ddagger}\right]\end{array}$ & $158(74)$ [0.1646] & 160 (73) [reference] & $163(72)$ [0.062] & $169(71)[<0.001]$ & $151(68)[<0.001]$ & $153(68)[<0.001]$ & $139(70)[<0.001]$ \\
\hline
\end{tabular}

BMI: body mass index; mCER: measured 24-h urine creatinine excretion; pCER: predicted 24-h urine creatinine excretion. ${ }^{*}: p$-value for mean difference determined by two sample $t$-test. 
The sex-specific mCER ranges had the lowest mean urine sodium excretion $(137( \pm 66) \mathrm{mmol} / 24-\mathrm{h})$ and the creatinine index $\geq 0.7$ method had the highest mean urine sodium excretion $(169( \pm 74) \mathrm{mmol} / 24-\mathrm{h})$. Compared to the $158( \pm 76) \mathrm{mmol} / 24-\mathrm{h}$ mean urine sodium of the self-reported no missed urine (the reference method), all methods had a statistically different mean measured 24-h urine sodium ( $p<$ at the significance level of 0.05) (Table 1). The Kernel density plots suggest sex-specific mCER ranges had a lower mean and less dispersion of urine sodium excretion than the other methods, but all other methods had a similar dispersion of urinary sodium excretion (Figure 1). Women had higher mean 24-h urine sodium excretions than men (Table 1) in samples included by self-reported no missed urine and sex-specific mCER ranges (Table 1). Men consistently had higher 24-h urine creatinine concentrations than women. The sex-specific 24 -h urine creatinine concentrations did not vary across methods except for the sex-specific mCER ranges, which included samples with lower 24-h urine creatinine concentrations for both sexes (Table 2).

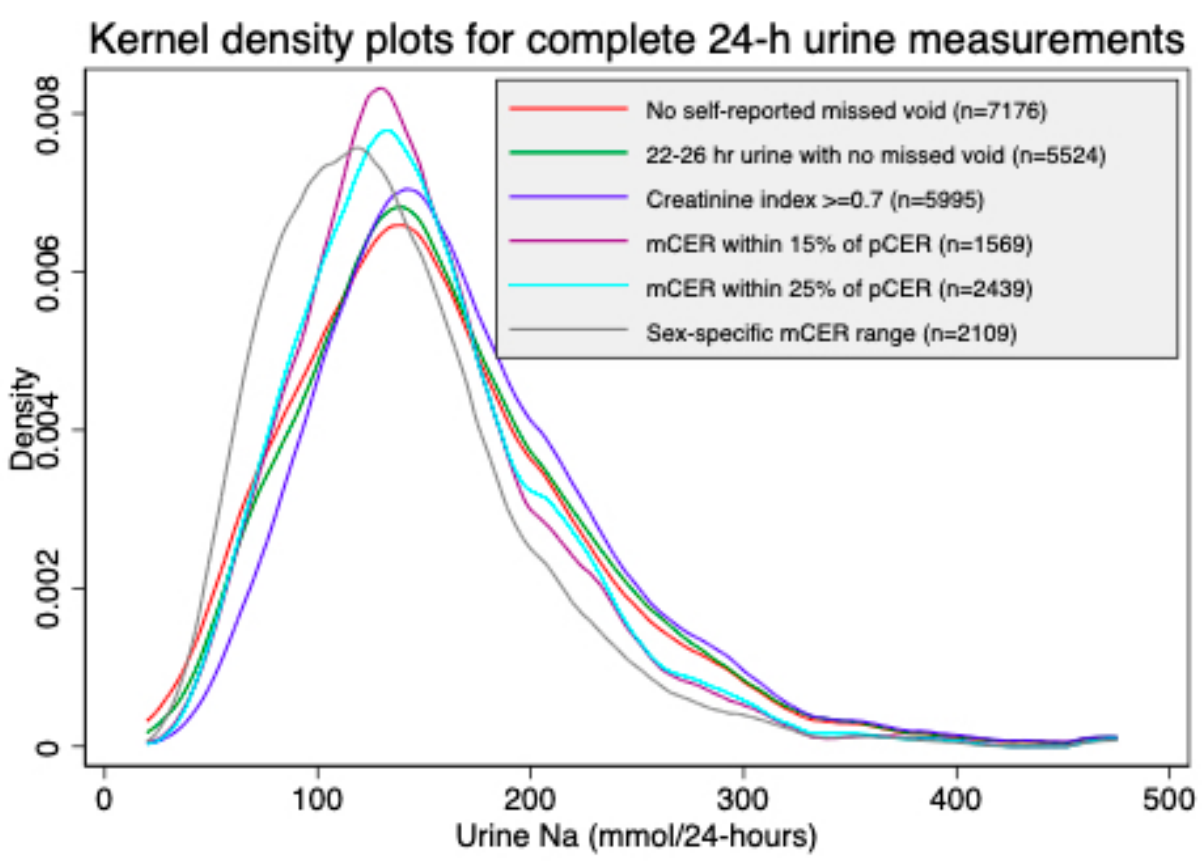

Figure 1. Kernel density plots of measured 24-h urine sodium excretion based on different methods of calculating complete 24-h urine samples. mCER: measured 24-h urine creatinine. excretion; pCER: predicted 24-h urine creatinine excretion.

Table 2. Sex-specific 24-h urine volume and 24-h urine creatinine concentrations (mg/dL) from different methods of evaluating the completeness of 24 -h urine samples.

\begin{tabular}{ccccc}
\hline \multirow{2}{*}{ Methods of Evaluating 24-h Urine Samples } & \multicolumn{2}{c}{ Urine Volume in L, Median (IQR) } & \multicolumn{2}{c}{ Urine Creatinine Concentration } \\
\cline { 2 - 5 } in mg/dL, Median (IQR)
\end{tabular}

mCER: measured 24-h urine creatinine excretion; pCER: predicted 24-h urine creatinine excretion. 
For all methods, the covariates-adjusted restricted cubic spline plots illustrated an initial steep positive sodium-SBP and sodium-DBP relationship (Figure 2). Afterward, we found a less steep positive sodium-SBP relationship for self-reported no missed voids, 22-26 h collection with no missed voids, creatinine index $\geq 0.7$, and sex-specific $\mathrm{mCER}$ ranges; but a plateaued sodium-SBP relationship for the two Kawasaki formula-based methods (Figure 2). In contrast, a negative sodium-DBP relationship was observed after the initial steep positive relationship for the self-reported no missed voids, 22-26 h collection with no missed voids, and the two Kawasaki formula-based methods; and a plateaued sodium-DBP relationship for the creatinine index $\geq 0.7$ and sex-specific $m C E R$ range methods (Figure 2 ). The sex-specific covariates-adjusted restricted cubic spline plots suggested a consistent positive sodium-SBP relationship across all methods for women but a varied sodium-SBP relationship for men (Figure 3).
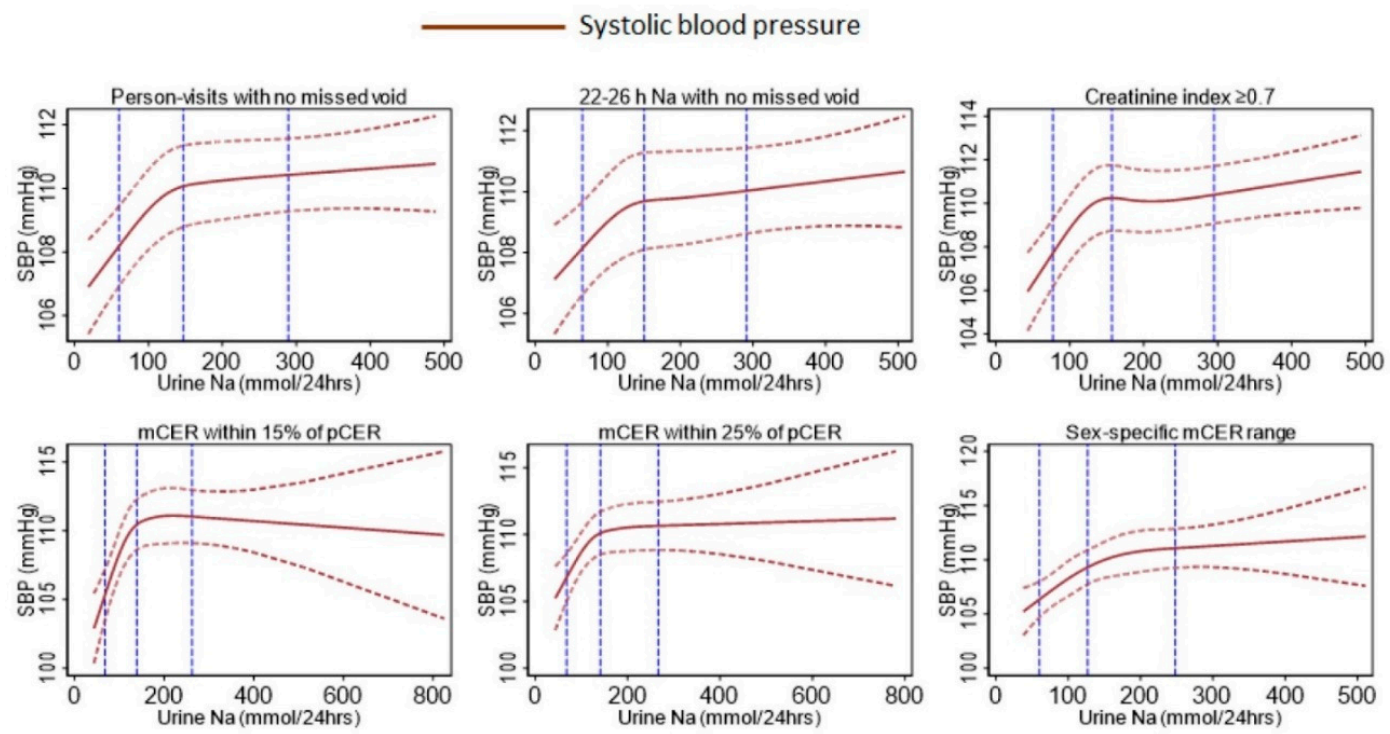

- Diastolic blood pressure
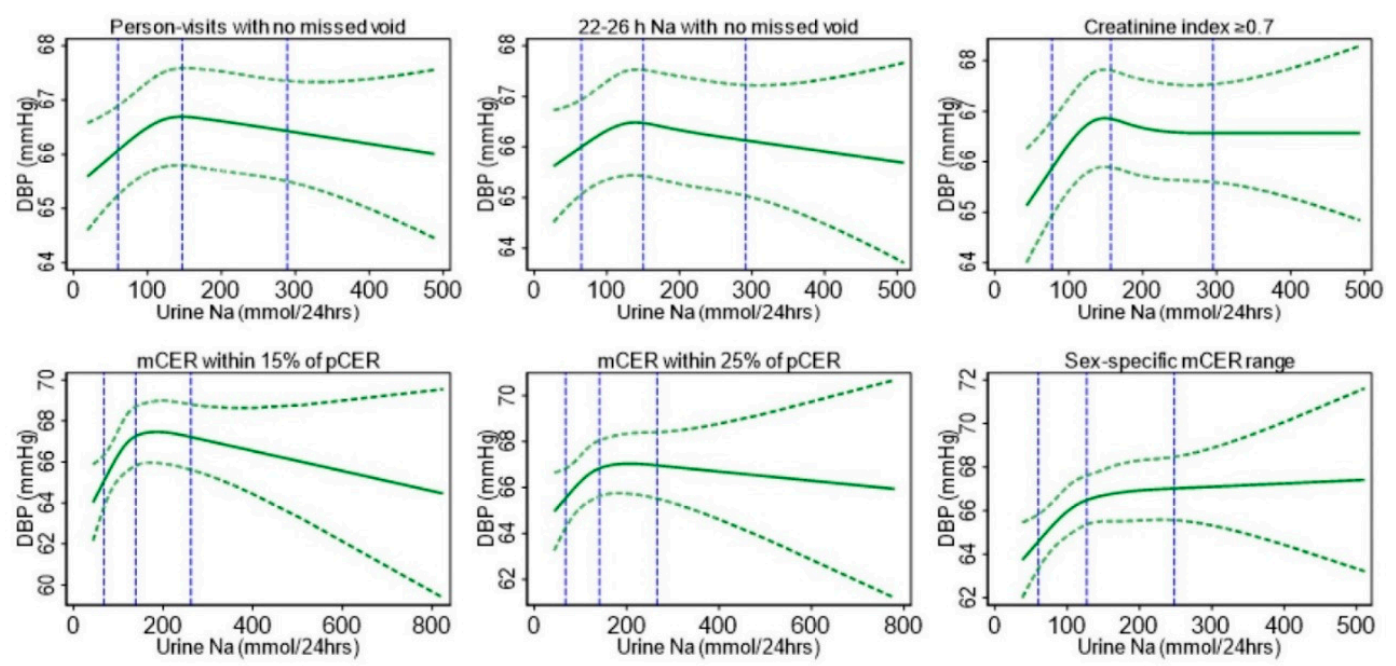

Figure 2. Restricted cubic spline pots of urinary sodium and blood pressure relationship from different method of evaluating the completeness of 24-h urine collection. Models were adjusted for age, sex, BMI, smoking status, physical activity, sleep, alcohol consumption, religion, and household wealth. Vertical dotted blue dotted lines indicate the 5th, 50th, and 95th percentile distribution of urine sodium excretion. mCER: measured 24-h urine creatinine excretion; pCER: predicted 24-h urine creatinine excretion. 

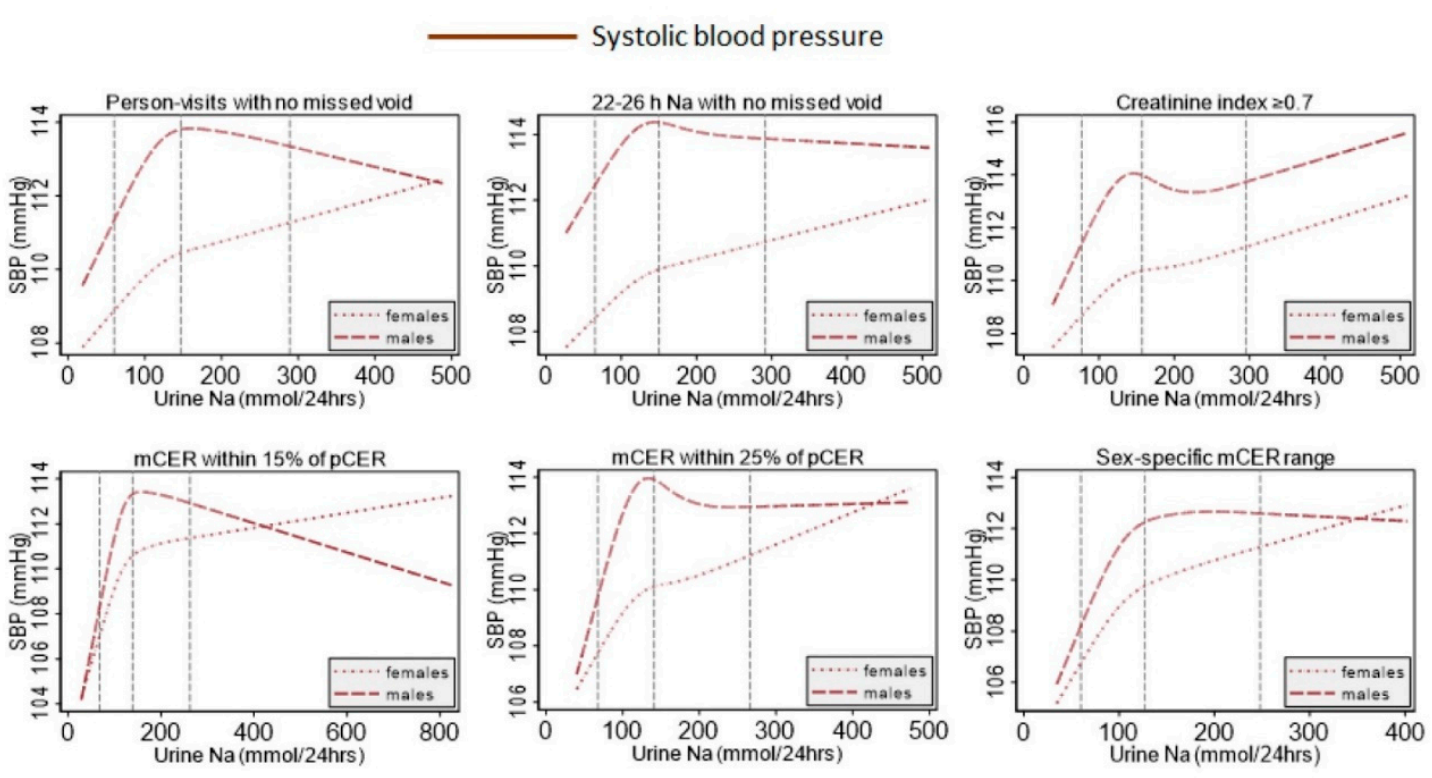

Diastolic blood pressure
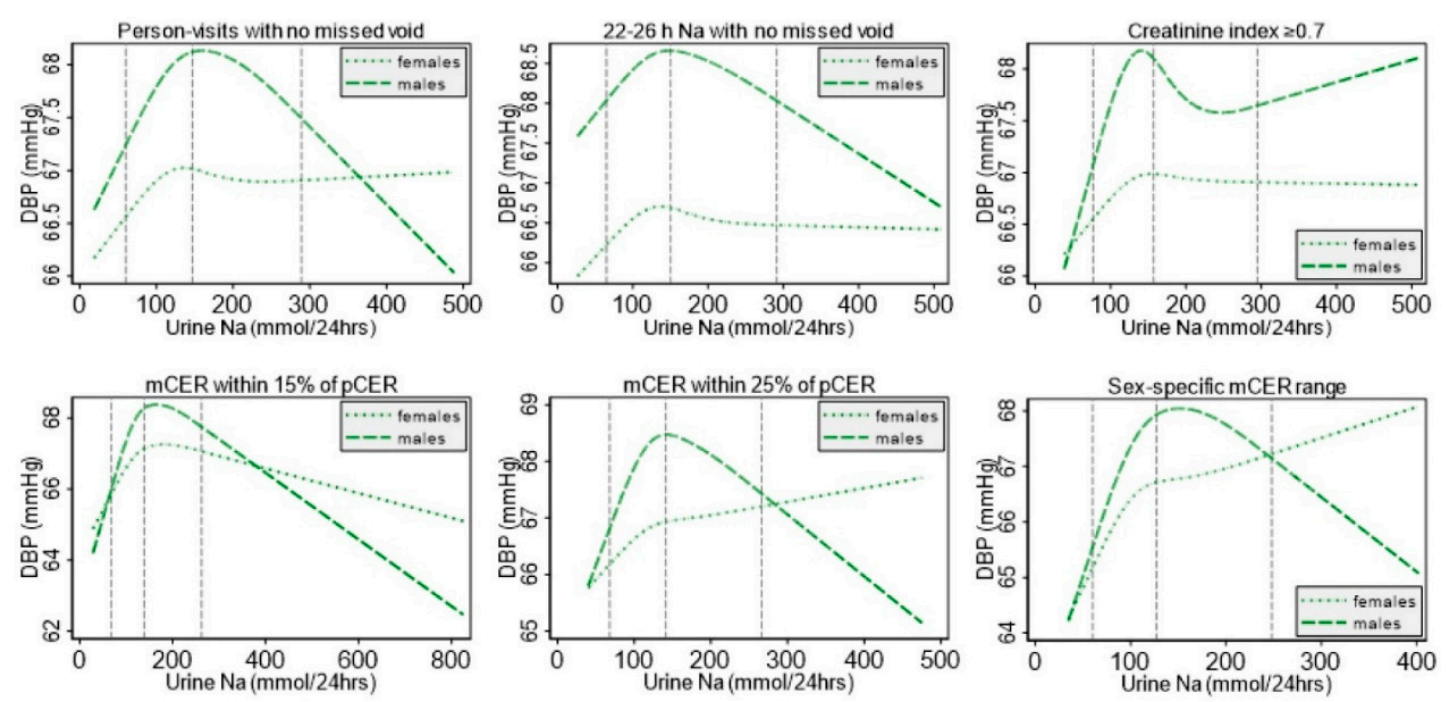

Figure 3. Restricted cubic spline plots of the sex-specific relationship between urinary sodium and blood pressure from different methods of evaluating the completeness of 24-h urine collection. Models were adjusted for age, sex, BMI, smoking status, physical activity, sleep, alcohol consumption, religion, and household wealth. Vertical dotted blue dotted lines indicate the 5th, 50th, and 95th percentile distribution of urine sodium excretion. mCER: measured 24-h urine creatinine excretion; pCER: predicted 24-h urine creatinine excretion.

In the fully adjusted model using tertiles of 24-h urinary sodium, there was a graded increase in $\mathrm{BP}$ with increases in sodium. Compared to the tertile 1 person-visits, those in tertile 3 had 3.36 (95\% CI: 1.75, 4.96) $\mathrm{mmHg}$ higher SBP for the sex-specific mCER ranges, 2.97 (95\% CI: 1.48, 4.46) $\mathrm{mmHg}$ higher SBP for the mCER within 15\% of Kawasaki predicted creatinine, and 1.55 (95\% CI: 0.37, 2.72) $\mathrm{mmHg}$ higher SBP for the mCER within $25 \%$ of Kawasaki predicted creatinine (Table 3). In the full-adjusted model, compared to the tertile 1 person-visits, those in tertile 3 had 1.66 (95\% CI: $0.66,2.66) \mathrm{mmHg}$ higher DBP for the sex-specific mCER ranges, 1.20 (95\% CI: 0.25, 2.15) $\mathrm{mmHg}$ higher DBP for the mCER within 15\% of Kawasaki predicted creatinine, and 0.41 (95\% CI: 0.01, 0.81) mmHg higher DBP for the creatinine index $\geq 0.7$ method. A linear trend of the sodium-SBP relationship was found for 
all methods, but a linear trend of the sodium-DBP relationship was found only for the sex-specific mCER range, mCER within $15 \%$ of Kawasaki predicted creatinine, and creatinine index $\geq 0.7$ methods (Table 3).

In the full-adjusted model, each $100 \mathrm{mmol} / 24-\mathrm{h}$ increase in urinary sodium was associated with 0.68 (95\% CI: $0.27,1.08) \mathrm{mmHg}$ higher SBP and 0.09 (95\% CI: $-0.17,0.36) \mathrm{mmHg}$ higher DBP for the creatinine index $\geq 0.7$ method, 0.98 (95\% CI: $-0.07,2.02) \mathrm{mmHg}$ change in SBP and 0.30 (95\% CI: -0.09 , 0.70) $\mathrm{mmHg}$ higher DBP for mCER within 15\% Kawasaki predicted urine creatinine, 0.87 (95\% CI: $0.07,1.67) \mathrm{mmHg}$ higher SBP and 0.29 (95\% CI: -0.12, 0.71) mmHg higher DBP for mCER within $25 \%$ Kawasaki predicted urine creatinine, and 1.96 (95\% CI: 0.93, 2.99) mmHg higher SBP and 0.88 (95\% CI: $0.16,1.59) \mathrm{mmHg}$ higher DBP for the sex-specific mCER range (Table 4).

Table 3. Association between the tertile of urinary sodium and blood pressure.

\begin{tabular}{|c|c|c|c|c|c|c|c|c|}
\hline \multirow{2}{*}{$\begin{array}{l}\text { Methods for } \\
\text { Complete 24-h } \\
\text { Urine Assessment }\end{array}$} & \multicolumn{4}{|c|}{ Systolic BP (mmHg) } & \multicolumn{4}{|c|}{ Diastolic BP (mmHg) } \\
\hline & Tertile 1 & Tertile 2 & Tertile 3 & $\begin{array}{l}p \text {-Value } \\
\text { for Trend }\end{array}$ & Tertile 1 & Tertile 2 & Tertile 3 & $\begin{array}{l}p \text {-Value } \\
\text { for Trend }\end{array}$ \\
\hline \multicolumn{9}{|c|}{ No self-reported missed voids } \\
\hline Model 1 & Ref & $\begin{array}{c}0.60(0.01 \\
1.19)\end{array}$ & $\begin{array}{c}0.98(0.36 \\
1.60)\end{array}$ & 0.002 & Ref & $\begin{array}{c}-0.04(-0.42 \\
0.35)\end{array}$ & $\begin{array}{c}0.14(-0.24 \\
0.52)\end{array}$ & 0.463 \\
\hline Model 2 & Ref & $\begin{array}{c}0.75(0.15 \\
1.35)\end{array}$ & $\begin{array}{c}1.14(0.57 \\
1.71)\end{array}$ & $<0.001$ & Ref & $\begin{array}{c}0.01(-0.34 \\
0.36)\end{array}$ & $\begin{array}{c}0.12(-0.26 \\
0.49)\end{array}$ & 0.545 \\
\hline Model 3 & Ref & $\begin{array}{c}0.73(0.14 \\
1.33)\end{array}$ & $\begin{array}{c}1.15(0.58 \\
1.72)\end{array}$ & $<0.001$ & Ref & $\begin{array}{c}-0.01(-0.35 \\
0.33)\end{array}$ & $\begin{array}{c}0.13(-0.25 \\
0.50)\end{array}$ & 0.508 \\
\hline \multicolumn{9}{|c|}{ 22-26 h urine samples with no missed voids } \\
\hline Model 1 & Ref & $\begin{array}{c}0.62(0.06 \\
1.17)\end{array}$ & $\begin{array}{c}1.03(0.35 \\
1.70)\end{array}$ & 0.003 & Ref & $\begin{array}{c}0.01(-0.48 \\
0.48)\end{array}$ & $\begin{array}{c}0.12(-0.37 \\
0.61)\end{array}$ & 0.625 \\
\hline Model 2 & Ref & $\begin{array}{c}0.75(0.22 \\
1.27)\end{array}$ & $\begin{array}{c}1.09(0.44 \\
1.73)\end{array}$ & 0.001 & Ref & $\begin{array}{c}0.03(-0.39 \\
0.45)\end{array}$ & $\begin{array}{c}0.01(-0.46 \\
0.47)\end{array}$ & 0.984 \\
\hline Model 3 & Ref & $\begin{array}{c}0.74(0.22 \\
1.26)\end{array}$ & $\begin{array}{c}1.10(0.47 \\
1.73)\end{array}$ & $<0.001$ & Ref & $\begin{array}{c}0.02(-0.39 \\
0.43)\end{array}$ & $\begin{array}{c}0.01(-0.44 \\
0.46)\end{array}$ & 0.963 \\
\hline \multicolumn{9}{|c|}{ Creatinine index $\geq 0.7 \&$ no missed voids } \\
\hline Model 1 & Ref & $\begin{array}{c}1.13(0.55 \\
1.72)\end{array}$ & $\begin{array}{c}1.31(0.71 \\
1.92)\end{array}$ & $<0.001$ & Ref & $\begin{array}{c}0.51(0.12 \\
0.89)\end{array}$ & $\begin{array}{c}0.53(0.18 \\
0.88)\end{array}$ & 0.003 \\
\hline Model 2 & Ref & $\begin{array}{c}1.24(0.65 \\
1.84) \\
\end{array}$ & $\begin{array}{c}1.33(0.75 \\
1.91)\end{array}$ & $<0.001$ & Ref & $\begin{array}{c}0.52(0.15 \\
0.90)\end{array}$ & $\begin{array}{c}0.41(0.02 \\
0.80)\end{array}$ & 0.037 \\
\hline Model 3 & Ref & $\begin{array}{c}1.21(0.62 \\
1.81)\end{array}$ & $\begin{array}{c}1.33(0.74 \\
1.92)\end{array}$ & $<0.001$ & Ref & $\begin{array}{c}0.50(0.12 \\
0.87)\end{array}$ & $\begin{array}{l}0.41(0.01 \\
0.81)\end{array}$ & 0.047 \\
\hline \multicolumn{9}{|c|}{ mCER within $15 \%$ of Kawasaki predicted daily creatinine } \\
\hline Model 1 & Ref & $\begin{array}{c}2.21(0.82 \\
3.60)\end{array}$ & $\begin{array}{c}2.56(1.03 \\
4.09)\end{array}$ & 0.001 & Ref & $\begin{array}{c}0.95(-0.01 \\
1.91)\end{array}$ & $\begin{array}{c}1.38(0.41 \\
2.35)\end{array}$ & 0.005 \\
\hline Model 2 & Ref & $\begin{array}{c}2.49(1.02 \\
3.95)\end{array}$ & $\begin{array}{c}2.96(1.45 \\
4.46)\end{array}$ & 0.001 & Ref & $\begin{array}{c}0.84(-0.13 \\
1.81)\end{array}$ & $\begin{array}{c}1.88(0.24 \\
2.14)\end{array}$ & 0.014 \\
\hline Model 3 & Ref & $\begin{array}{c}2.49(1.02 \\
3.97)\end{array}$ & $\begin{array}{c}2.97(1.48 \\
4.46)\end{array}$ & $<0.001$ & Ref & $\begin{array}{c}0.87(-0.11 \\
1.84)\end{array}$ & $\begin{array}{c}1.20(0.25 \\
2.15)\end{array}$ & 0.013 \\
\hline \multicolumn{9}{|c|}{ mCER within $25 \%$ of Kawasaki predicted daily creatinine } \\
\hline Model 1 & Ref & $\begin{array}{c}0.63(-0.44 \\
1.69)\end{array}$ & $\begin{array}{c}1.41(0.17 \\
2.64)\end{array}$ & 0.025 & Ref & $\begin{array}{c}0.18(-0.51 \\
0.88)\end{array}$ & $\begin{array}{c}0.72(-0.12 \\
1.56)\end{array}$ & 0.095 \\
\hline Model 2 & Ref & $\begin{array}{c}0.80(-0.28 \\
1.88)\end{array}$ & $\begin{array}{c}1.54(0.34 \\
2.74)\end{array}$ & 0.012 & Ref & $\begin{array}{c}0.08(-0.63 \\
0.80)\end{array}$ & $\begin{array}{c}0.46(-0.40 \\
1.31)\end{array}$ & 0.295 \\
\hline Model 3 & Ref & $\begin{array}{c}0.79(-0.28 \\
1.85)\end{array}$ & $\begin{array}{c}1.55(0.37 \\
2.72)\end{array}$ & 0.010 & Ref & $\begin{array}{c}0.08(-0.65 \\
0.80)\end{array}$ & $\begin{array}{c}0.46(-0.40 \\
1.31)\end{array}$ & 0.295 \\
\hline
\end{tabular}


Table 3. Cont.

\begin{tabular}{|c|c|c|c|c|c|c|c|c|}
\hline \multirow{2}{*}{$\begin{array}{l}\text { Methods for } \\
\text { Complete 24-h } \\
\text { Urine Assessment }\end{array}$} & \multicolumn{4}{|c|}{ Systolic BP (mmHg) } & \multicolumn{4}{|c|}{ Diastolic BP (mmHg) } \\
\hline & Tertile 1 & Tertile 2 & Tertile 3 & $\begin{array}{l}p \text {-Value } \\
\text { for Trend }\end{array}$ & Tertile 1 & Tertile 2 & Tertile 3 & $\begin{array}{l}p \text {-Value } \\
\text { for Trend }\end{array}$ \\
\hline \multicolumn{9}{|c|}{$\mathrm{mCER} 15-25 \mathrm{mg} / \mathrm{kg} / 24 \mathrm{~h}$ for men and $10-20 \mathrm{mg} / \mathrm{kg} / 24 \mathrm{~h}$ for women } \\
\hline Model 1 & Ref & $\begin{array}{c}2.31(1.09 \\
3.52)\end{array}$ & $\begin{array}{c}3.83(2.19 \\
5.46)\end{array}$ & $<0.001$ & Ref & $\begin{array}{c}1.59(0.81 \\
2.38)\end{array}$ & $\begin{array}{c}2.29(1.33 \\
3.25)\end{array}$ & $<0.001$ \\
\hline Model 2 & Ref & $\begin{array}{c}2.01(0.88 \\
3.15)\end{array}$ & $\begin{array}{c}3.34(1.76 \\
4.91)\end{array}$ & $<0.001$ & Ref & $\begin{array}{c}1.23(0.45 \\
2.02)\end{array}$ & $\begin{array}{c}1.62(0.63 \\
2.61)\end{array}$ & 0.001 \\
\hline Model 3 & Ref & $\begin{array}{c}2.01(0.85 \\
3.16)\end{array}$ & $\begin{array}{c}3.36(1.75 \\
4.96)\end{array}$ & $<0.001$ & Ref & $\begin{array}{c}1.23(0.45 \\
2.02)\end{array}$ & $\begin{array}{c}1.66(0.66 \\
2.66)\end{array}$ & 0.001 \\
\hline
\end{tabular}

No self-reported missed voids-urine sodium tertile 1: $<121.9 \mathrm{mmol} /$ day; tertile $2:>=121.9$ to $<175.8 \mathrm{mmol} /$ day; tertile 3: $>=175.8 \mathrm{mmol} /$ day. $22-26 \mathrm{~h}$ urine samples with no missed voids - urine sodium tertile $1:<126.4 \mathrm{mmol} /$ day; tertile 2: $>=126.4$ to $<178.9 \mathrm{mmol} /$ day; tertile $3:>=178.9 \mathrm{mmol} /$ day. Creatinine index $\geq 0.7$-urine sodium tertile 1: $<133.7 \mathrm{mmol} /$ day; tertile $2:>=133.7$ to $<186.1 \mathrm{mmol} /$ day; tertile $3:>=186.1 \mathrm{mmol} /$ day. $\mathrm{mCER}$ within $15 \%$ of Kawasaki predicted daily creatinine — urine sodium tertile $1:<119.4 \mathrm{mmol} /$ day; tertile $2:>=119.4$ to $<162.9 \mathrm{mmol} /$ day; tertile 3 : $>=162.9 \mathrm{mmol} /$ day. $\mathrm{mCER}$ within $25 \%$ of Kawasaki predicted daily creatinine: $<119.7 \mathrm{mmol} /$ day; tertile $2:>=119.7$ to $<166.1 \mathrm{mmol} /$ day; tertile 3: >=166.1 mmol/day. Sex-specific mCER ranges-urine sodium tertile 1: $<104.2 \mathrm{mmol} /$ day; tertile 2: $>=104.2$ to $<151 \mathrm{mmol} /$ day; tertile $3:>=151 \mathrm{mmol} /$ day. mCER: measured $24-\mathrm{h}$ urine creatinine excretion; pCER: predicted 24-h urine creatinine excretion. Model 1: Unadjusted; Model 2: adjusted for age, sex, and body mass index (BMI); Model 3: additionally, adjusted for physical activities, smoking status, alcohol consumption, sleep hours, religion, and household wealth. ${ }^{*} p$-value $<0.05$ suggestive of a linear trend.

Table 4. Difference in mean systolic BP (mmHg) for $100 \mathrm{mmol}$ or $2300 \mathrm{mg}$ per 24-h increase in 24-h urine sodium.

\begin{tabular}{|c|c|c|c|}
\hline Methods for Complete 24-h Urine Assessment & $\begin{array}{c}\text { Model } 1 \\
\beta(95 \% \text { CI })\end{array}$ & $\begin{array}{c}\text { Model } 2 \\
\beta(95 \% \text { CI })\end{array}$ & $\begin{array}{c}\text { Model } 3 \\
\beta(95 \% \text { CI })\end{array}$ \\
\hline \multicolumn{4}{|c|}{ Systolic blood pressure } \\
\hline No self-reported missed voids $(n=7176)$ & $0.57(0.28,0.86)$ & $0.64(0.34,0.94)$ & $0.64(0.34,0.94)$ \\
\hline $22-26 \mathrm{~h}$ urine samples with no missed voids $(\mathrm{n}=5524)$ & $0.54(0.21,0.87)$ & $0.53(0.19,0.87)$ & $0.54(0.21,0.87)$ \\
\hline Creatinine index $\geq 0.7$ and no missed voids $(\mathrm{n}=5995)$ & $0.67(0.29,1.06)$ & $0.68(0.28,1.09)$ & $0.68(0.27,1.08)$ \\
\hline $\begin{array}{c}\text { mCER within } 15 \% \text { of Kawasaki pCER and no missed } \\
\text { voids }(n=1569)\end{array}$ & $0.86(-0.11,1.84)$ & $0.97(-0.08,2.02)$ & $0.98(-0.07,2.02)$ \\
\hline $\begin{array}{c}\text { mCER within } 25 \% \text { of Kawasaki pCER and no missed } \\
\text { voids }(\mathrm{n}=2439)\end{array}$ & $0.81(0.05,1.58)$ & $0.87(0.06,1.67)$ & $0.87(0.07,1.67)$ \\
\hline $\begin{array}{l}\mathrm{mCER} 15-25 \mathrm{mg} / \mathrm{kg} / 24 \mathrm{~h}(\mathrm{men}) \text { and } 10-20 \mathrm{mg} / \mathrm{kg} / 24 \mathrm{~h} \\
\text { (women), and no missed voids }(\mathrm{n}=2109)\end{array}$ & $2.25(1.10,3.39)$ & $1.94(0.90,2.98)$ & $1.96(0.93,2.99)$ \\
\hline \multicolumn{4}{|c|}{ Diastolic blood pressure } \\
\hline No self-reported missed voids $(\mathrm{n}=7176)$ & $0.06(-0.15,0.27)$ & $0.02(-0.18,0.23)$ & $0.03(-0.18,0.24)$ \\
\hline 22-26 h urine samples with no missed voids $(n=5524)$ & $0.04(-0.22,0.30)$ & $\begin{array}{l}-0.04(-0.30 \\
0.21)\end{array}$ & $\begin{array}{l}-0.04(-0.30 \\
0.22)\end{array}$ \\
\hline Creatinine index $\geq 0.7$ and no missed voids $(\mathrm{n}=5995)$ & $0.16(-0.09,0.40)$ & $0.09(-0.16,0.35)$ & $0.09(-0.17,0.36)$ \\
\hline $\begin{array}{c}\text { mCER within } 15 \% \text { of Kawasaki pCER and no missed } \\
\text { voids }(n=1569)\end{array}$ & $0.38(-0.07,0.83)$ & $0.29(-0.1,0.69)$ & $0.30(-0.09,0.70)$ \\
\hline $\begin{array}{c}\text { mCER within } 25 \% \text { of Kawasaki pCER and no missed } \\
\text { voids }(\mathrm{n}=2439)\end{array}$ & $0.41(-0.03,0.84)$ & $0.29(-0.12,0.71)$ & $0.29(-0.12,0.71)$ \\
\hline $\begin{array}{l}\mathrm{mCER} 15-25 \mathrm{mg} / \mathrm{kg} / 24 \mathrm{~h}(\mathrm{men} \text { ) and } 10-20 \mathrm{mg} / \mathrm{kg} / 24 \mathrm{~h} \\
\text { (women), and no missed voids }(\mathrm{n}=2109)\end{array}$ & $1.30(0.53,2.08)$ & $0.85(0.13,1.56)$ & $0.88(0.16,1.59)$ \\
\hline
\end{tabular}

mCER: measured 24-h urine creatinine excretion; pCER: predicted 24-h urine creatinine excretion. Model 1: Unadjusted; Model 2: adjusted for age, sex, and body mass index (BMI); Model 3: additionally, adjusted for physical activities, smoking status, alcohol consumption, sleep hours, religion, and household wealth. 


\section{Discussion}

Twenty-four-hour urine collection is considered the most accurate method to assess dietary sodium intake. However, incomplete collection of urine samples is very common, particularly in population-based studies. Researchers across the world have used various methods to exclude incomplete 24-h urine samples. Our study demonstrates that the use of different methods led to significant differences in mean 24-h urine sodium excretion and altered the association of excreted sodium with BP, including the shape, magnitude, and significance levels. These differences were observed in all modeling strategies we assessed. Furthermore, we found a sex-specific difference in the urine sodium excretion and BP relationship. The different methods of assessing incomplete 24-h urine samples resulted in the exclusion of different proportions of urine collections. Additionally, the different methods resulted in differences in which specific collections were included or excluded. Likely, the indirect methods we evaluated may not effectively exclude all of the true incomplete 24-h urine samples and, at the same time, some complete 24-h urine samples may have been excluded in this Bangladeshi population. Since we did not have the gold standard PABA method, we are unable to determine which method performed the best.

The creatinine index $\geq 0.7$ excludes samples with low urine creatinine, which is analogous to the exclusion of samples based on urine creatinine below $15 \mathrm{mg} / \mathrm{kg} / 24-\mathrm{h}$ [11]. The other three creatinine-based methods exclude samples with both low or high urine creatinine levels; therefore, they were more restrictive methods that included fewer urine samples. Of the creatinine-based methods, the trio of creatinine index $\geq 0.7$, mCER within $25 \%$, and mCER within $15 \%$ of Kawasaki predicted urine creatinine had some consistencies in terms of selecting complete 24-h urine samples because the latter method includes a sub-sample of urine collections selected by the former method. Therefore, mCER within $25 \%$ of Kawasaki predicted urine creatinine was a more restrictive approach than the creatinine index $\geq 0.7$, and a further restrictive method was the mCER within $15 \%$ of Kawasaki predicted urine creatinine. Nevertheless, the sex-specific mCER ranges had considerable discrepancies with the other three creatinine-based methods. Nearly one in four samples included by the sex-specific ranges were excluded by creatinine index $\geq 0.7$, and one in three samples included by mCER within $15 \%$ of Kawasaki predicted urine creatinine were excluded by the sex-specific mCER range. Such discrepancy raises concerns about the effectiveness of creatinine-based methods to evaluate the completeness of 24-h urine samples among the Bangladeshi population.

Creatinine-based methods exclude samples if measured urine creatinine excretion levels are outside of the sex-specific physiological ranges; however, these methods consider neither participants' age nor muscle mass, protein intake, and other variables influencing measured urine creatinine excretion. These methods were developed from a small number of participants; therefore, they may not reflect population-level characteristics and variability. For example, the creatinine index $\geq 0.7$ was evaluated in 541 participants, the Kawasaki equation of completeness evaluation was developed from 487 participants, and the sex-specific mCER ranges was evaluated in 104 participants [11,12,29]. Significant variability of urinary creatinine excretion was observed in National Health and Nutrition Examination Survey participants across age groups, with young adults showing greater variation [36]. Therefore, creatinine-based methods may not be sensitive for a large population where participants are recruited from a wide age range. A systematic review suggested that the creatinine index $\geq 0.7$ had a sensitivity of $49 \%$ and specificity of $88 \%$ when compared with the gold standard direct para-aminobenzoic acid excretion [12,37]. That means one in two samples selected by creatinine index $\geq 0.7$ were not a complete 24 -h urine sample. The sensitivity and specificity of the creatinine-based methods are likely different in our study population and that might have resulted in inaccurate inclusion of many incomplete 24-h urine samples in our analyses. Erroneous inclusion or exclusion of 24-h urine samples may explain the difference in the shape and magnitude of the urine sodium excretion and blood pressure relationship.

We identified a plateaued relationship between the urine sodium and systolic blood pressure relationship above the 50th percentile distributions of urine sodium. Randomized controlled trials, 
in contrast, show a mostly linear relationship between dietary sodium intake and blood pressure, with potentially a steeper relationship at sodium intake below $100 \mathrm{mmol} /$ day. Residual confounding, with physical activity and other variables that co-relate with blood pressure and sodium intake may explain some of the plateauing. One of the other possibilities includes the presence of blood pressure-lowering minerals (e.g., potassium, magnesium) in the brackish water. In other analyses reported elsewhere [14], we identified that drinking brackish water is associated with not only a high intake of sodium but also higher intakes of magnesium, calcium, and potassium. We identified that urinary magnesium and calcium were associated with lower blood pressure, whereas urine sodium was associated with higher blood pressure. Further sex-specific plots identified such a plateaued or declined urine sodium and systolic blood pressure relationship above the 50th percentile distribution of urine sodium was in men, while women consistently had a positive upward relationship. The sex-specific differences can be attributable to factors such as sex-specific variation in the sodium intake and blood pressure association [38-40] and the sex-specific difference in muscle mass and urinary creatinine excretion. Creatinine is derived from skeletal muscle at a constant rate and excreted by the glomerulus. Creatinine is neither reabsorbed nor metabolized by the kidney tubular cells. South Asians have low muscle mass [40]; hence, creatinine-based methods may need adjustments for south Asians with low muscle mass. Males have a higher variation of urinary creatinine than females [41]; therefore, more urine samples of males are likely to be excluded by the creatinine-based indirect methods of 24-h completeness evaluation. In South Asian culture, males may have more variability in the collection of 24-h urine sample protocol since they work outside compared to females who stay at home. This could have led to the inclusion of a relatively lower proportion of samples of male person-visits across methods of complete 24-h sample determination.

The sex-specific mCER ranges method excluded samples when measured urinary creatinine was low or high. The mean urine sodium excretion for the sex-specific mCER ranges was the lowest of all the methods, suggesting sex-specific mCER ranges included more data-points with low urine sodium excretion. Since the urine sodium and blood pressure relationship plots had a steep slope in the early segment of the plots, it also explains a higher magnitude of urine sodium and blood pressure association for sex-specific mCER ranges in linear analyses.

The study population had high sodium intake, compared to the WHO-recommended daily intakes of less than $2000 \mathrm{mg} /$ day or $87 \mathrm{mmol} /$ day. Only $8 \%$ of person-visits had urine sodium within $1500 \mathrm{mg} /$ day or $65.2 \mathrm{mmol} /$ day, the recommended daily intake by the Dietary Approaches to Stop Hypertension (DASH) study. We consistently found a steep slope of the urine sodium and blood pressure relationship curve in the spline plots' initial sections. This suggests reducing daily sodium intake below the recommended daily intakes may reduce the population's mean blood pressure markedly, albeit a small portion of person visits were within the recommended urine sodium excretion.

Our study has several important strengths and limitations. We had cross-sectional sodium exposure and blood pressure outcome data for several visits for the same individual. Many hemodynamic changes associated with salt load occur within $24 \mathrm{~h}$. Therefore, blood pressure, along with sodium intake, measurement is particularly advantageous instead of a time lag between sodium intake and blood pressure measurement. We had a relatively large sample size that provided enough power for analyses, even after excluding samples by more restrictive methods of complete 24-h collection evaluation. Inclusion of participant-, household-, and community-level random intercepts largely captured time non-variant unmeasured confounders at each level and therefore minimized the bias. The use of Omron ${ }^{\circledR}$ HEM-907 rather than using different instruments would have provided standardized blood pressure measurements, reducing the measurement errors in blood pressure measurement. Twenty-four-hour urine collection studies are recommended to incorporate estimation of the completeness of 24-h urine in a subsample using para-aminobenzoic acid [10], a gold standard approach to determining completeness. Lacking that component, we were unable to compare the creatinine-based and self-reported methods of completeness evaluation with para-aminobenzoic acid. Hence, we are not sure which urine samples are complete and which are not. Weather variables, 
particularly ambient temperature, rainfall, and humidity, affect blood pressure by influencing the peripheral resistance of skin vasculature [42-44]. We did not measure these weather variables, which precludes us from controlling for them. Nevertheless, future analyses need to be implemented after retrieving the weather data from the local weather station to see how weather variables influence the urine sodium and blood pressure relationship. Temperature and physical activities also influence the rate of sweating and sodium excretion [45], however, the sodium excretion through sweat decreases markedly among acclimatized individuals within weeks $[46,47]$. High sweat flow may overwhelm the ability to reabsorb sodium from sweat. Therefore, sweating can potentially lead to decreased urinary sodium excretion, particularly for men, who may not have been fully acclimatized during the study [23]. Hence, bias due to sodium excretion in sweat may also have influenced our analyses. Our study was limited to the Bangladeshi population predominantly from the southwest coastal region and may not be generalizable to other populations. Blood pressure has a diurnal variation, with morning blood pressure usually being higher than that measured around noon or afternoon [48]. We were unable to control for diurnal variation of blood pressure since we did not collect the exact time of blood pressure measurement, which may have introduced some bias in our estimates.

\section{Conclusions}

Our findings suggest that the results of epidemiological studies evaluating the health effects of sodium intake can be influenced by different methods of evaluating the completeness of 24-h urine samples. It is therefore possible that other studies using 24-h urine collections without considering the completeness of urine samples may also have provided altered and unreliable associations between sodium and health outcomes. Low-quality epidemiological studies lead to more debates causing controversy and could impact public health policy on sodium reduction. It is vital to ensure the 24-h urine collections are complete in epidemiological studies, preferably using para-aminobenzoic acid to assess the completeness of the collected urine samples, so as to provide robust evidence on sodium intake and health outcomes. Further research on the evaluation of indirect methods of complete 24-h urine samples is needed, particularly to compare them with para-aminobenzoic acid excretion.

Author Contributions: Conceptualization, N.R.C.C., F.J.H.; methodology, A.M.N., F.J.H., N.R.C.C.; formal analysis, A.M.N.; writing—original draft preparation, A.M.N.; writing—review and editing, F.J.H., M.R., K.M.V.N., N.R.C.C.; supervision, K.M.V.N.; funding acquisition, A.M.N., M.R. All authors have read and agreed to the published version of the manuscript.

Funding: This research was funded by the Wellcome Trust, UK through an award under the Our Planet, Our Health Programme (Grant \# 106871/Z/15/Z). The corresponding author's time was partly supported by the National Heart, Lung, and Blood Institute funded T32 training grant (Grant \# T32 HL130025).

Acknowledgments: We acknowledge with gratitude the commitment of the Wellcome Trust, UK for supporting the research. We are grateful to the participants in the study for their support and cooperation. Icddr,b is also grateful to the Governments of Bangladesh, Canada, Sweden and the UK for providing core and unrestricted support. We are grateful to colleagues at the University of Dhaka and UNICEF, Bangladesh for their assistance to conduct the study.

Conflicts of Interest: The authors declare no conflict of interest.

\section{References}

1. Forouzanfar, M.H.; Afshin, A.; Alexander, L.T.; Anderson, H.R.; Bhutta, Z.A.; Biryukov, S.; Brauer, M.; Burnett, R.; Cercy, K.; Charlson, F.J.; et al. Global, regional, and national comparative risk assessment of 79 behavioural, environmental and occupational, and metabolic risks or clusters of risks, 1990-2015: A systematic analysis for the Global Burden of Disease Study 2015. Lancet 2016, 388, 1659-1724. [CrossRef]

2. Karppanen, H.; Karppanen, P.; Mervaala, E. Why and how to implement sodium, potassium, calcium, and magnesium changes in food items and diets? J. Hum. Hypertens. 2005, 19, S10-S19. [CrossRef]

3. Cogswell, M.E.; Mugavero, K.; Bowman, B.A.; Frieden, T.R. Dietary sodium and cardiovascular disease risk-Measurement matters. N. Engl. J. Med. 2016, 375, 580. [CrossRef] 
4. He, F.J.; MacGregor, G.A. Salt reduction lowers cardiovascular risk: Meta-analysis of outcome trials. Lancet 2011, 378, 380-382. [CrossRef]

5. He, F.J.; Tan, M.; Ma, Y.; MacGregor, G.A. Salt Reduction to Prevent Hypertension and Cardiovascular Disease: JACC State-of-the-Art Review. J. Am. Coll. Cardiol. 2020, 75, 632-647. [CrossRef]

6. Tan, M.; He, F.J.; MacGregor, G.A. Salt and cardiovascular disease in PURE: A large sample size cannot make up for erroneous estimations. J. Renin-Angiotensin-Aldosterone Syst. 2018, 19. [CrossRef]

7. Powles, J.; Fahimi, S.; Micha, R.; Khatibzadeh, S.; Shi, P.; Ezzati, M.; Engell, R.E.; Lim, S.S.; Danaei, G.; Mozaffarian, D. Global, regional and national sodium intakes in 1990 and 2010: A systematic analysis of $24 \mathrm{~h}$ urinary sodium excretion and dietary surveys worldwide. BMJ Open 2013, 3, e003733. [CrossRef]

8. Santos, J.A.; Sparks, E.; Thout, S.R.; McKenzie, B.; Trieu, K.; Hoek, A.; Johnson, C.; McLean, R.; Arcand, J.; Campbell, N.R. The Science of Salt: A global review on changes in sodium levels in foods. J. Clin. Hypertens. 2019, 21, 1043-1056. [CrossRef]

9. World Health Organization. The SHAKE Technical Package for Salt Reduction; WHO: Geneva, Switzerland, 2016.

10. Campbell, N.R.; He, F.J.; Tan, M.; Cappuccio, F.P.; Neal, B.; Woodward, M.; Cogswell, M.E.; McLean, R.; Arcand, J.; MacGregor, G. The International Consortium for Quality Research on Dietary Sodium/Salt (TRUE) position statement on the use of 24-hour, spot, and short duration ( $<24$ hours) timed urine collections to assess dietary sodium intake. J. Clin. Hypertens. 2019, 21, 700-709. [CrossRef]

11. Wielgosz, A.; Robinson, C.; Mao, Y.; Jiang, Y.; Campbell, N.R.; Muthuri, S.; Morrison, H. The impact of using different methods to assess completeness of 24-hour urine collection on estimating dietary sodium. J. Clin. Hypertens. 2016, 18, 581-584. [CrossRef]

12. John, K.A.; Cogswell, M.E.; Campbell, N.R.; Nowson, C.A.; Legetic, B.; Hennis, A.J.; Patel, S.M. Accuracy and usefulness of select methods for assessing complete collection of 24-hour urine: A systematic review. J. Clin. Hypertens. 2016, 18, 456-467. [CrossRef]

13. Nowson, C.A.; Lim, K.; Campbell, N.R.; O'Connell, S.L.; He, F.J.; Daly, R.M. Impact of fractional excretion of sodium on a single morning void urine collection as an estimate of 24-hour urine sodium. J. Clin. Hypertens. 2019, 21, 1763-1770. [CrossRef]

14. Naser, A.M.; Rahman, M.; Unicomb, L.; Doza, S.; Gazi, M.S.; Alam, G.R.; Karim, M.R.; Uddin, M.N.; Khan, G.K.; Ahmed, K.M.; et al. Drinking water salinity, urinary macro-mineral excretions, and blood pressure in the southwest coastal population of Bangladesh. J. Am. Heart Assoc. 2019, 8, e012007. [CrossRef]

15. Khan, A.E.; Ireson, A.; Kovats, S.; Mojumder, S.K.; Khusru, A.; Rahman, A.; Vineis, P. Drinking water salinity and maternal health in coastal Bangladesh: Implications of climate change. Environ. Health Perspect. 2011, 119, 1328-1332. [CrossRef]

16. Khan, A.E.; Scheelbeek, P.F.D.; Shilpi, A.B.; Chan, Q.; Mojumder, S.K.; Rahman, A.; Haines, A.; Vineis, P. Salinity in drinking water and the risk of (pre) eclampsia and gestational hypertension in coastal Bangladesh: A case-control study. PLoS ONE 2014, 9, e108715. [CrossRef]

17. Scheelbeek, P.F.; Chowdhury, M.A.; Haines, A.; Alam, D.S.; Hoque, M.A.; Butler, A.P.; Khan, A.E.; Mojumder, S.K.; Blangiardo, M.A.; Elliott, P.; et al. Drinking water salinity and raised blood pressure: Evidence from a cohort study in coastal Bangladesh. Environ. Health Perspect. 2017, 125. [CrossRef]

18. Talukder, M.R.R.; Rutherford, S.; Phung, D.; Islam, M.Z.; Chu, C. The effect of drinking water salinity on blood pressure in young adults of coastal Bangladesh. Environ. Pollut. 2016, 248-254. [CrossRef]

19. Sultana, S.; Ahmed, K.; Mahtab-Ul-Alam, S.; Hasan, M.; Tuinhof, A.; Ghosh, S.; Rahman, M.; Ravenscroft, P.; Zheng, Y. Low-cost aquifer storage and recovery: Implications for improving drinking water access for rural communities in coastal Bangladesh. J. Hydrol. Eng. 2015, 20, B5014007. [CrossRef]

20. Atikul Islam, M.; Sakakibara, H.; Karim, M.R.; Sekine, M. Potable water scarcity: Options and issues in the coastal areas of Bangladesh. J. Water Health 2013, 11, 532-542. [CrossRef]

21. Naser, A.M.; Rahman, M.; Unicomb, L.; Doza, S.; Anand, S.; Chang, H.; Luby, S.; Clasen, T.; Narayan, K. Comparison of Urinary Sodium and Blood Pressure Relationship from the Spot Versus 24-Hour Urine Samples. J. Am. Heart Assoc. 2019, 8, e013287. [CrossRef]

22. Naser, A.M.; Unicomb, L.; Doza, S.; Ahmed, K.M.; Rahman, M.; Uddin, M.N.; Quraishi, S.B.; Selim, S.; Shamsudduha, M.; Burgess, W.; et al. Stepped-wedge cluster-randomised controlled trial to assess the cardiovascular health effects of a managed aquifer recharge initiative to reduce drinking water salinity in southwest coastal Bangladesh: Study design and rationale. BMJ Open 2017, 7, e015205. [CrossRef] 
23. Naser, A.M.; Doza, S.; Rahman, M.; Unicomb, L.; Ahmed, K.M.; Anand, S.; Selim, S.; Shamsudduha, M.; Narayan, K.; Chang, H.; et al. Consequences of access to water from managed aquifer recharge systems for blood pressure and proteinuria in south-west coastal Bangladesh: A stepped-wedge cluster-randomized trial. Int. J. Epidemiol. 2020. [CrossRef] [PubMed]

24. Naser, A.M.; Rahman, M.; Unicomb, L.; Parvez, S.M.; Islam, S.; Doza, S.; Khan, G.K.; Ahmed, K.M.; Anand, S.; Luby, S.P.; et al. Associations of drinking rainwater with macro-mineral intake and cardiometabolic health: A pooled cohort analysis in Bangladesh, 2016-2019. npj Clean Water 2020, 1-11. [CrossRef]

25. Gunnsteinsson, S.; Labrique, A.B.; West Jr, K.P.; Christian, P.; Mehra, S.; Shamim, A.A.; Rashid, M.; Katz, J.; Klemm, R.D. Constructing indices of rural living standards in Northwestern Bangladesh. J. Health Popul. Nutr. 2010, 28, 509.

26. Taussky, H.H.; Kurzmann, G. A microcolorimetric determination of creatine in urine by the Jaffe reaction. J. Biol. Chem. 1954, 208, 853-861.

27. Kawasaki, T.; Itoh, K.; Uezono, K.; Sasaki, H. A simple method for estimating $24 \mathrm{~h}$ urinary sodium and potassium excretion from second morning voiding urine specimen in adults. Clin. Exp. Pharmacol. Physiol. 1993, 20, 7-14. [CrossRef]

28. Walser, M. Creatinine excretion as a measure of protein nutrition in adults of varying age. J. Parenter. Enter. Nutr. 1987, 11, 73S-78S. [CrossRef]

29. Kawasaki, T.; Uezono, K.; Itoh, K.; Ueno, M. Prediction of 24-hour urinary creatinine excretion from age, body weight and height of an individual and its application. Jpn. J. Public Health 1991, 38, 567-574.

30. Orsini, N.; Greenland, S. A procedure to tabulate and plot results after flexible modeling of a quantitative covariate. Stata J. 2011, 11, 1. [CrossRef]

31. Harrell, F.E., Jr. Regression Modeling Strategies: With Applications to Linear Models, Logistic and Ordinal Regression, and Survival Analysis; Springer: Berlin, Germany, 2015.

32. Smith, Z.; Knight, T.; Sahota, P.; Kernohan, E.; Baker, M. Dietary patterns in Asian and Caucasian men in Bradford: Differences and implications for nutrition education. J. Hum. Nutr. Diet. 1993, 6, 323-333. [CrossRef]

33. Aggarwal, B.; Makarem, N.; Shah, R.; Emin, M.; Wei, Y.; St-Onge, M.P.; Jelic, S. Effects of inadequate sleep on blood pressure and endothelial inflammation in women: Findings from the American Heart Association go red for women strategically focused Research network. J. Am. Heart Assoc. 2018, 7, e008590. [CrossRef]

34. Wilcox, R. Trimming and winsorization. Encycl. Biostat. 2005, 8. [CrossRef]

35. Hubert, L.J. The use of orthogonal polynomials for trend analysis. Am. Educ. Res. J. 1973, 10, $241-244$. [CrossRef]

36. Barr, D.B.; Wilder, L.C.; Caudill, S.P.; Gonzalez, A.J.; Needham, L.L.; Pirkle, J.L. Urinary creatinine concentrations in the US population: Implications for urinary biologic monitoring measurements. Environ. Health Perspect. 2005, 113, 192-200. [CrossRef]

37. De Keyzer, W.; Huybrechts, I.; Dekkers, A.L.; Geelen, A.; Crispim, S.; Hulshof, P.J.; Andersen, L.F.; Řehůřková, I.; Ruprich, J.; Volatier, J.-L.; et al. Predicting urinary creatinine excretion and its usefulness to identify incomplete $24 \mathrm{~h}$ urine collections. Br. J. Nutr. 2012, 108, 1118-1125. [CrossRef]

38. He, J.; Gu, D.; Chen, J.; Jaquish, C.E.; Rao, D.C.; Hixson, J.E.; Chen, J.-C.; Duan, X.; Huang, J.-F.; Chen, C.-S.; et al. Gender difference in blood pressure responses to dietary sodium intervention in the GenSalt study. J. Hypertens. 2009, 27, 48. [CrossRef]

39. Mill, J.G.; Baldo, M.P.; Molina, M.D.C.B.; Schmidt, M.I.; Barreto, S.M.; Chor, D.; Griep, R.H.; Matos, S.M.; Ribeiro, A.L.P.; Duncan, B.B.; et al. Sex-specific patterns in the association between salt intake and blood pressure: The ELSA-Brasil study. J. Clin. Hypertens. 2019, 21, 502-509. [CrossRef]

40. Pechère-Bertschi, A.; Burnier, M. Female sex hormones, salt, and blood pressure regulation. Am. J. Hypertens. 2004, 17, 994-1001. [CrossRef]

41. Lermen, D.; Bartel-Steinbach, M.; Gwinner, F.; Conrad, A.; Weber, T.; von Briesen, H.; Kolossa-Gehring, M. Trends in characteristics of 24-h urine samples and their relevance for human biomonitoring studies-20 years of experience in the German Environmental Specimen Bank. Int. J. Hyg. Environ. Health 2019, 222, 831-839. [CrossRef]

42. Aubinière-Robb, L.; Jeemon, P.; Hastie, C.E.; Patel, R.K.; McCallum, L.; Morrison, D.; Walters, M.; Dawson, J.; Sloan, W.; Muir, S.; et al. Blood pressure response to patterns of weather fluctuations and effect on mortality. Hypertension 2013, 62, 190-196. [CrossRef] 
43. Brook, R.D.; Weder, A.B.; Rajagopalan, S. "Environmental hypertensionology" the effects of environmental factors on blood pressure in clinical practice and research. J. Clin. Hypertens. 2011, 13, 836-842. [CrossRef]

44. Modesti, P.A. Season, temperature and blood pressure: A complex interaction. Eur. J. Intern. Med. 2013, 24, 604-607. [CrossRef] [PubMed]

45. Bates, G.P.; Miller, V.S. Sweat rate and sodium loss during work in the heat. J. Occup. Med. Toxicol. $2008,3,4$. [CrossRef] [PubMed]

46. Buono, M.J.; Kolding, M.; Leslie, E.; Moreno, D.; Norwood, S.; Ordille, A.; Weller, R. Heat acclimation causes a linear decrease in sweat sodium ion concentration. J. Therm. Biol. 2018, 71, 237-240. [CrossRef] [PubMed]

47. Périard, J.; Racinais, S.; Sawka, M.N. Adaptations and mechanisms of human heat acclimation: Applications for competitive athletes and sports. Scand. J. Med. Sci. Sports 2015, 25, 20-38. [CrossRef]

48. White, W.B. Importance of blood pressure control over a 24-hour period. J. Manag. Care Pharm. 2007, 13 (Suppl. B), 34-39. [CrossRef]

(C) 2020 by the authors. Licensee MDPI, Basel, Switzerland. This article is an open access article distributed under the terms and conditions of the Creative Commons Attribution (CC BY) license (http://creativecommons.org/licenses/by/4.0/). 